\title{
Conocimiento de las abejas nativas sin aguijón y cambio generacional entre los mayas lacandones de Nahá, Chiapas
}

\author{
Native Stingless Bee's Knowledge and \\ Generational Change among the Lacandon \\ Maya of Nahá, Chiapas
}

Leonardo Ernesto Ulises Contreras Cortés

Facultad de Ciencias Sociales, Universidad Autónoma de Chiapas, México

Amparo Vázquez García

Universidad Intercultural del Estado de Puebla, México

Elda Miriam Aldasoro Maya

El Colegio de la Frontera Sur, Unidad Villahermosa, México

Consejo Nacional de Ciencia y Tecnología, México

JoRGE MÉRIDA RIVAS

El Colegio de la Frontera Sur, Unidad San Cristóbal de Las Casas, México

\begin{abstract}
Resumen: Los mayas lacandones están asentados en la selva lacandona desde el siglo xvI; su experiencia y conocimiento les permite conocer y aprovechar distintos elementos naturales, entre ellos, las Abejas Nativas Sin Aguijón (ANSA). El presente trabajo registró las especies de ANSA y el conocimiento diferenciado que sobre ellas tienen los jefes de familia en Nahá, Chiapas. De 2015 a 2018, con colaboradores clave y a través de colectas entomológicas, se determinaron las especies de ANSA que identifica la población local en el Área de Protección Flora y Fauna de Nahá (APFFN). Posteriormente se aplicó un cuestionario a 68 jefes de familia, todos hombres, para estimar el grado de conocimiento y los usos que les dan a las abejas nativas sin aguijón, de acuerdo a estratos edad. En total se registraron 15 especies de abejas nativas, entre ellas se extrae miel principalmente de cuatro: Tetragonisca angustula (Ajyus), Scaura argyrea (K'amas kap'), Plebeia frontalis (Ak chip kap) y Melipona solani (Jach K'ojo'). Registramos un conocimiento diferenciado de los jefes de familia en las 15 especies ANSA, a partir de su comportamiento, morfología y lugares donde
\end{abstract}


viven. La tendencia generalizada es a la pérdida de conocimiento, acentuándose en los jóvenes. Las causas se vinculan con el debilitamiento de prácticas sociales de transmisión de conocimiento, como disminución de la recolección y falta de manejo de colmenas en sus solares; aunado a ello, se suma la llegada de productos que sustituyen la miel y la cera, ofertados a precios más bajos.

Palabras clave: mayas lacandones; abejas nativas sin aguijón; miel; pérdida de conocimiento tradicional.

AвSTRACT: The Lacandon Maya were settled in the Lacandon forest since the 17th century; their experience and knowledge allow them to know and manage different natural elements, like the Native Stingless Bees (NSB). The present research registered the species of NSB and the differentiated knowledge about them in Nahá, Chiapas. From 2015 to 2018, through work with key collaborators and entomological collections, the species of NSB identified by the local population of the Flora and Fauna Protection Area of Nahá (FFPAN) were determined. Later a questionnaire was applied to 68 heads of family, all males, to estimate the level of knowledge they have and the uses they give to the native stingless bees, according to age ranges. A total of 15 species of native bees were registered, of which honey is obtained mainly from four: Tetragonisca angustula (Ajyus), Scaura argyrea (K'amas kap'), Plebeia frontalis (Ak chip kap) y Melipona solani (Jach K'ojo'). We registered a differentiated knowledge of the heads of family in Nahá about the 15 species of NSB based on their behavior, morphology and the places where they live. The general tendency is the loss of knowledge, and it is accentuated among the young people. The causes are related to the weakening of the transmission's mechanisms of knowledge, the decrease of the recollection activities and the absence of management of colonies in their homegardens, as well as the arrival of products that substitute honey and wax, offered at lower prices.

KeYworDs: Lacandon Maya; native stingless bees; honey; loss of traditional knowledge.

RECEPCIÓN: 30 de junio de 2019.

ACEPTACIÓN: 17 de septiembre de 2019.

Dor: https://doi.org/10.19130/iifl.ecm.2020.56.2.0008

\section{Introducción}

Las abejas de la tribu Meliponini (Familia Apidae) son insectos cuyo número de especies a nivel mundial alcanzan al menos 500; éstas habitan en regiones tropicales y subtropicales en todo el mundo y se caracterizan por tener un aguijón atrofiado. En el continente americano existen alrededor de 400 especies distribuidas desde Argentina hasta el norte de México (Michener, 2007; 2013). En México, Ayala (1999) y Ayala, González y Engel (2013) señalan que las especies registradas alcanzan 46, 12 de las cuales son endémicas. Para el estado de Chia- 
pas, Arnold et al. (2018a; 2018b) reportan 34, mientras que Contreras (2018) señala la existencia de 32.

El manejo de las abejas nativas sin aguijón (en adelante, ANSA) por poblaciones locales del centro y suroeste de México está registrado desde la época prehispánica (Sotelo, Guerrero y Álvarez, 2012). Actualmente la presencia de la meliponicultura se ubica en diferentes regiones del país. En la península de Yucatán, los mayas manejan las abejas sin aguijón desde la época prehispánica (Echazarreta et al., 1997; González, 2012; Ayala y Engel, 2013). Otras regiones de manejo de las ANSA en México son la sierra norte de Puebla (Cuetzalan), las Huastecas (sobre todo la Potosina), el Totonacapan en Veracruz, la Sierra de Atoyac en Guerrero (González, 2012; Padilla et al., 2014; Patlán et al., 2014), en Tabasco (Murillo, 1984; Cano, Martínez y Balboa, 2013; Aldasoro, Arnold y Burguete, 2015; Chan et al., 2019), Oaxaca (Arnold et al., 2018a), Michoacán (Reyes, Camou y Gómez, 2016) y Chiapas (Guzmán et al., 2011).

Actualmente, a pesar de existir la meliponicultura en diversos estados, su propagación es paulatina debido a la introducción de la abeja europea (Apis mellife$\mathrm{ra}$ ) cuya producción de miel es mayor que la de los meliponinos (Arnold et al., 2018a). Aunque la meliponicultura es una práctica realizada desde hace muchos años por pueblos originarios, la pérdida del conocimiento sobre su manejo se debe a cambios socioculturales expresados por la falta de interés, sobre todo en la población joven, la deforestación provocada por la expansión de actividades extensivas más redituables, como la ganadería, y el empleo de pesticidas (Villanueva, Roubik y Colli, 2005: 35-36; Pat et al., 2018: 228-229). A pesar de ello Arnold et al. (2018a) documentaron diversas iniciativas tanto de organizaciones sociales y civiles como de instituciones gubernamentales que difunden la meliponicultura como una actividad productiva y de conservación ambiental en diversos estados del país.

Es importante analizar la meliponicultura, no sólo como una actividad económica que puede proporcionar recursos a poblaciones locales, sino como un patrimonio biocultural de relevancia para el mantenimiento del ecosistema. En este sentido, Aldasoro, Arnold y Burguete (2015) y Aldasoro et al. (2016) proponen la meliponicultura como patrimonio biocultural debido a que es una actividad fundamental para el mantenimiento de la biodiversidad. Kremen et al. (2007) estiman que entre el 60 y $90 \%$ de las plantas necesitan un polinizador y las abejas son insectos que cumplen dicha función. Además, el desarrollo de prácticas socioculturales locales que promuevan la reproducción de la meliponicultura ayuda a mantener y propagar la biodiversidad de ambientes específicos. La relación humano-abeja nativa y su crianza implica un entramado de saberes y conocimientos que forman parte de la cosmovisión de los pueblos. Los diferentes tipos de meliponicultura existentes en México son parte de su diversidad biocultural. Por tanto, la conservación de este patrimonio biocultural es crucial. En esta investigación se tuvo como objetivos elaborar un inventario de las especies conocidas en la localidad y determinar quiénes aprovechan sus productos y para qué, de modo que ello per- 
mitirá formar una base para la implementación de estrategias de conservación de la diversidad biocultural en el Área de Protección de Flora y Fauna Nahá.

\section{Material y métodos}

El estudio se realizó en Nahá, municipio de Ocosingo, en el estado de Chiapas, México. Esta comunidad tiene una amplitud altitudinal que oscila de 780 a 1300 msnm y se localiza en una zona biogeográfica de transición donde se superponen características neárticas y neotropicales, condiciones que contribuyen a que sea un área con alta biodiversidad, pero al mismo tiempo un espacio ecológicamente frágil (Halffter, 1978; 1987; 2003). La población se localiza entre las coordenadas $16^{\circ} 56^{\prime} 41^{\prime \prime}$ y $17^{\circ} 00^{\prime} 42^{\prime \prime}$ de latitud norte y $91^{\circ} 32^{\prime} 52^{\prime \prime}$ a $91^{\circ} 37^{\prime} 43^{\prime \prime}$ de longitud oeste. El tipo de clima es $\mathrm{Aw}_{2}(\mathrm{w})$ (i') g, cálido subhúmedo con lluvias en verano (García, 2003) (Figura 1).

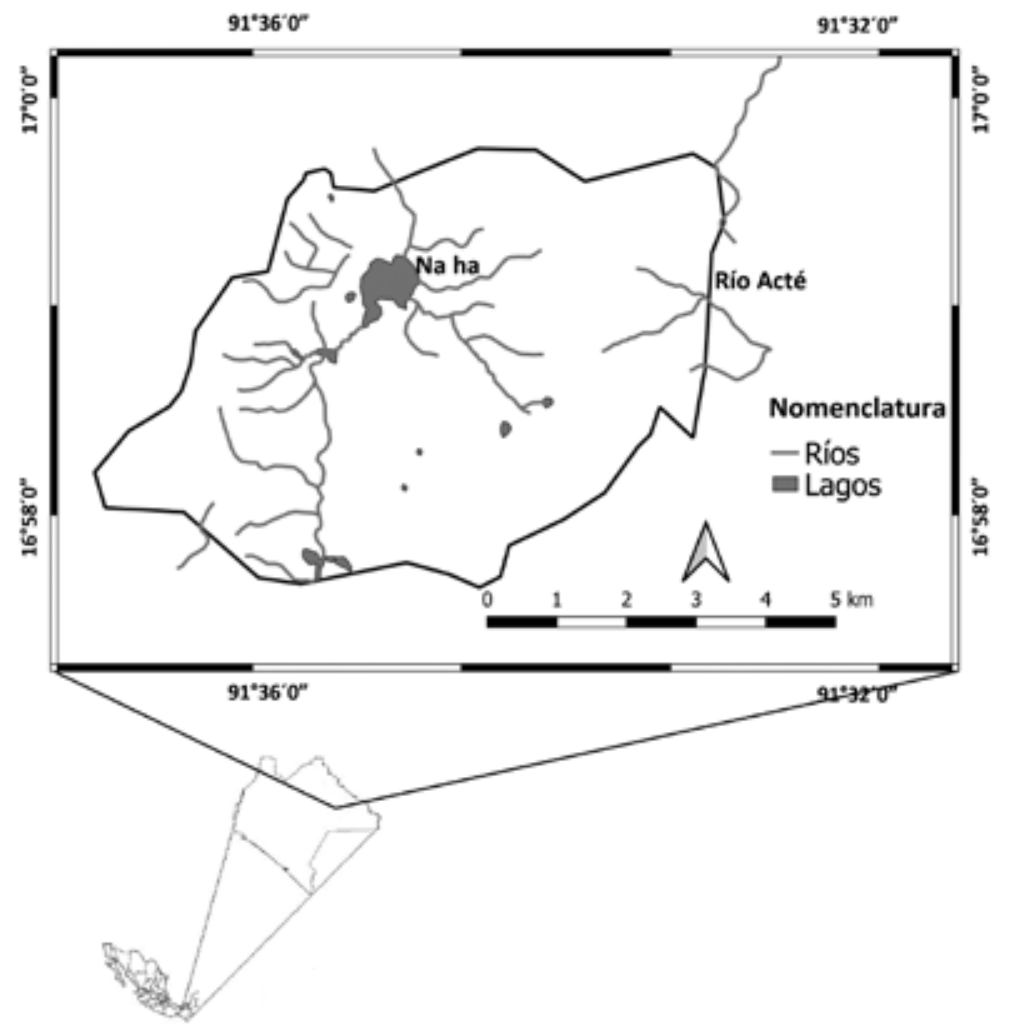

Figura 1. Mapa que muestra la localización de Nahá, Chiapas (Elaborado por Ulises Contreras). 
La vegetación de Nahá es diversa, existe selva alta, mediana y baja perennifolia, bosque mesófilo de montaña, bosque de coníferas, vegetación popal tular (Miranda y Hernández, 1963) y vegetación secundaria, que de acuerdo a Palacio et al. (2004) corresponde a los acahuales. Para el Instituto de Historia Natural (en adelante, IHN), en Nahá existen un total de 779 especies de plantas vasculares que pertenecen a 452 géneros de 116 familias ubicados en tres divisiones; el $51 \%$ de las especies se agrupan en las familias: Rubiaceae, Fabaceae, Orchidaceae, Bromeliaceae, Melastomataceae, Euphorbiaceae, Lauraceae, Araceae, Moraceae, Meliaceae y Arecaceae (IHN, 2000). De acuerdo con la Comisión Nacional de Áreas Protegidas (en adelante, ConANP) de las 3847 hectáreas el $85.55 \%$ corresponde a zonas de preservación, de uso tradicional, de asentamiento humano, de uso público y de aprovechamiento sustentable de los ecosistemas; el resto (14.45\%) son zonas de recuperación (CONANP, 2006: 91).

\section{Aspectos socio-culturales de Nahá}

Los actuales mayas lacandones pertenecen a la familia etnolingǘstica mayense y son el resultado de una fusión que tuvo lugar durante los siglos xvIII y xIx entre los sobrevivientes de comunidades exterminadas por los españoles y grupos de mayas fugitivos originarios del Petén guatemalteco y la península de Yucatán (Marion, 1999).

Diferentes etnografías dividen a los mayas lacandones, por su ubicación geográfica, en dos subgrupos: sur y norte (Baer y Merrifield, 1972; Boremanse, 1978; Eroza, 2006). Nahá pertenece a los mayas lacandones del norte, cuya cultura es más conservadora que la del sur; por ejemplo, la gente de mayor edad usa la vestimenta tradicional, las mujeres visten falda blanca y blusa de colores, mientras que los varones usan la túnica hecha de tela. Además, existe la costumbre de que cuando una pareja contrae nupcias, el hombre se muda a vivir durante un tiempo a la casa del suegro, para que éste observe y corrija las habilidades del yerno en prácticas como caza, pesca, recolección y siembra de milpa. Después de ese periodo, la pareja puede construir su propia casa. Este patrón de organización familiar permite asegurarse de que el nuevo jefe de familia tenga el suficiente conocimiento sobre el manejo y uso de la selva, y por otro lado fortalece la unidad familiar del suegro porque contará con una persona que le auxilie en todas las actividades cotidianas, estrategia que resuelve de forma parcial la falta de mano de obra masculina en las comunidades mayas lacandonas (Contreras y Mariaca, 2016).

En cuanto al número de habitantes, y de acuerdo a datos obtenidos por la Unidad Médica Rural, en 2014 la población de Nahá ascendía a 246 habitantes en un área de 3,847 ha, de los cuales 124 eran mujeres y 122 hombres. Nahá es una comunidad maya lacandona que al mismo tiempo tiene el estatus de Área Natural de Protección de Flora y Fauna (ANPFF) decretada en 1998. Esta categoría 
de protección implica la restricción de ciertas prácticas relacionadas con los bienes naturales, por ejemplo, el sistema agroecológico de milpa se modificó en su primera práctica pasando de roza-tumba-quema, a roza y quema.

En cuanto a la economía, las principales actividades son la agricultura de subsistencia, el aprovechamiento de sucesiones ecológicas, la caza — limitada a algunas especies, debido a las restricciones de la Norma 0059 de la Secretaría de Medio Ambiente y Recursos Naturales (SEMARNAT)—, la pesca, la recolección, las aves de traspatio, el turismo, la elaboración de artesanías y los ingresos derivados de los proyectos gubernamentales que proporcionan diversas instituciones (Contreras, Mariaca y Pérez, 2015).

\section{Obtención de datos etnoentomológicos}

La obtención de datos empezó a partir de octubre de 2015, previa autorización de la asamblea comunitaria y terminó en enero de 2018. Una primera etapa consistió en la aplicación de entrevistas abiertas a colaboradores clave, personas de edad avanzada y que tuvieran amplio conocimiento de la selva; con ello se pudo determinar los nombres de todas las especies de ANSA que conoce la población. Adicionalmente se realizaron colectas de cada una de las especies mencionadas, que fueron identificadas taxonómicamente por especialistas de la Colección de Abejas de El Colegio de la Frontera Sur. ${ }^{1}$ Con estos datos se hizo un listado de todas las abejas nativas sin aguijón que se conocen en la comunidad. Posteriormente se aplicó un cuestionario a 68 jefes de familia ( $80 \%$ del total existente), quienes tienen derecho otorgado por la comunidad para hacer uso y manejo de los bienes naturales de la reserva. Las preguntas se enfocaron a determinar dos grandes tópicos, por un lado los aspectos socioeconómicos de cada maya lacandón, como el sexo, la edad y la principal actividad, lo cual permitió conocer el perfil de las personas encuestadas, y por el otro, en aspectos relacionados con el reconocimiento de las especies de abejas nativas que reconocen, y en su caso si aprovechan algún producto que generen. Se analizaron las respuestas obtenidas y se realizó estadística descriptiva para el procesamiento de los datos.

\section{Resultados y discusión}

La información obtenida indica que la población encuestada se concentra en un rango de edad entre los 18 y 39 años (68\%), y que participan en mayor grado en proyectos gubernamentales y de organizaciones no gubernamentales (onGs) que aquellos que sobrepasan los 40 años de edad. En general, el 95.5\% de los encuestados se dedica al campo (agricultura, animales de traspatio, caza, pesca y

\footnotetext{
${ }^{1}$ ECOAB. Colección de Abejas de Ecosur.
} 
recolección). Es de notar que, de este porcentaje, el $41.2 \%$ son, además de agricultores, artesanos cuyo principal producto es la elaboración de flechas, además de formar parte de alguno de los dos grupos de socios de los centros ecoturísticos de Nahá y Pa'Cha'. También existen jefes de familia que tienen un empleo formal como policías y empleados del Área Natural Protegida (ANP) (11.9\%).

\section{Inventario de abejas nativas}

En total se registraron 15 especies de abejas nativas sin aguijón, con su nomenclatura local (Tabla 1). Sólo se capturaron especímenes que conocía la población, por ello es probable que puedan existir más ANSA en la reserva. Esencialmente se determinaron tres categorías de uso: alimenticio, artesanal y medicinal. El significado de cada actividad fue determinado con base en lo expresado por los mayas lacandones. El uso alimenticio se refiere al consumo de miel como endulzante, y como uso medicinal se refiere a la cura de ciertas afecciones. La miel usada como medicina procede de dos especies, Scaptotrigona pectoralis y Tetragonisca angustula, y se aplica para el tratamiento de enfermedades relacionadas con el sistema respiratorio. La cera de Trigona corvina y Trigona fulventris se mezcla con otros elementos y sirve como pegamento para adherir trozos de cuerda a flechas que son vendidas como artesanía.

\begin{tabular}{clc}
\hline & \multicolumn{1}{c}{ Nombre científico } & Nombre maya lacandón \\
1 & Cephalotrigona zexmeniae & K'anit \\
2 & Melipona solani & Jach k'ojo' o cojo \\
3 & Nannotrigona perilampoides & Bor kap \\
4 & Paratrigona opaca & At sats \\
5 & Partamona bilineata & K'e'k'an \\
6 & Plebeia frontalis & Ajchi'; Ak chip kap \\
7 & Plebeia llorentei & Mejen kap, yit mash \\
8 & Plebeia pulchra & Mejen kap, yit mash \\
9 & Scaptotrigona pectoralis & Ak tun kap'; kan sak (es rojo) \\
10 & Scaura argyrea & K'amas kap' \\
11 & Tetragona mayarum & Yus \\
12 & Tetragonisca angustula & Ajyus \\
13 & Trigona corvina & Ajxi'p' \\
14 & Trigona fulviventris & Murish kap, Pupus kap, Ajmur, mu kap \\
15 & Trigona nigerrima & Ajk'uk'is kap' \\
\hline
\end{tabular}

Tabla 1. Especies de abejas nativas que conoce la gente de Nahá, Chiapas (Trabajo de campo, octubre 2015 a enero 2018). 
De las 15 especies registradas que conocen los mayas lacandones, se consume miel de 12 de ellas, pero el consumo es más frecuente en sólo cuatro (Figura 2). La gente consume miel de las ANSA de acuerdo con sus características organolépticas; de las 12 mieles consumidas, las más apreciadas son Tetragonisca angustula (Ajyus), Scaura argyrea (K'amas kap'), Plebeia frontalis (Ajchi'; Ak chip kap) y Melipona solani (Jach k'ojo'), mientras que la miel de Trigona fulviventris (Murish kap, Pupus kap, Ajmur, mu kap) y Partamona bilineata (K'e'k'an) es la menos consumida porque es amarga, y en el caso de esta última, el comportamiento de la abeja que la produce es muy defensivo.

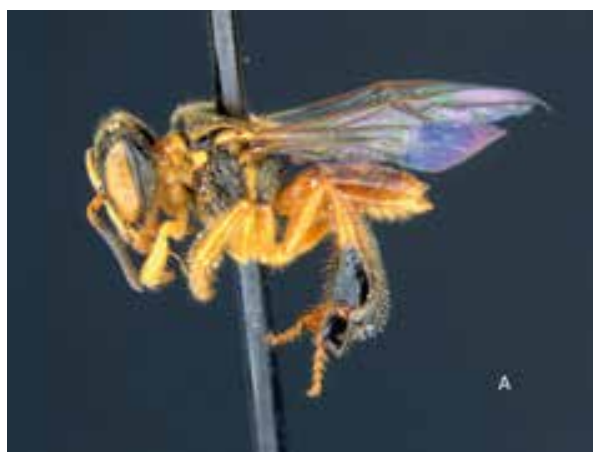

A

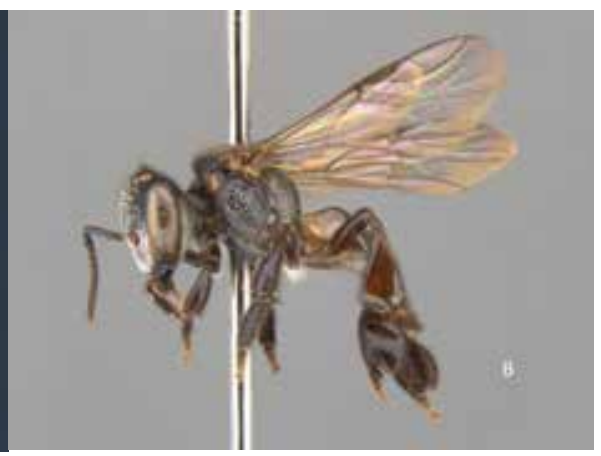

B

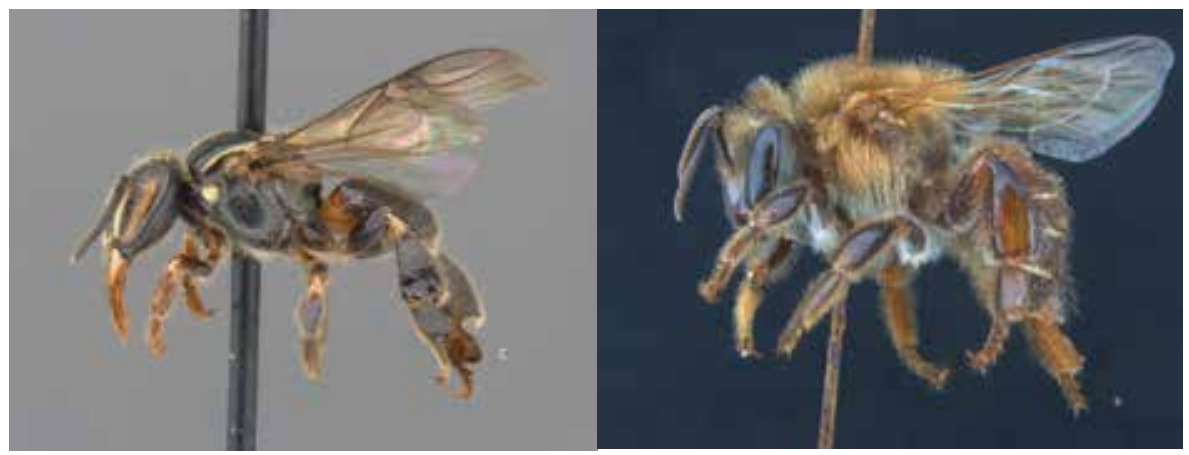

C

D

Figura 2. Las principales abejas que conocen los mayas lacandones.

A) Tetragonisca angustula (Ajyus), B) Scaura argyrea (K'amas kap') , C) Plebeia frontalis (Ak chip kap) y D) Melipona solani (Jach K'ojo') (Fotografías de Jorge Mérida).

\section{Conocimiento y uso local de cada especie}

Los saberes, conocimientos y prácticas de los mayas lacandones sobre el uso de los productos de las abejas que pueden aprovechar y sus diferentes nombres 
locales asignados para algunas de las especies (Tabla 1) indican indudablemente que las relaciones simbólicas, como la de planta/animal-humano, reflejan vínculos que van más allá de lo utilitario (Aldasoro, 2009).

A continuación, se describen cada una de las 15 especies de ANSA encontradas con las características observadas por los mayas lacandones; sólo se escribe el nombre científico, y la información señalada por la gente considera básicamente tres aspectos: etológico (por ejemplo, dóciles, defensivas), morfológico (color, tamaño, olor) y ecológico (dónde viven: en huertos, cerca de la comunidad, en la selva).

- Cephalotrigona zexmeniae (K'anit). Es una abeja dócil; de ésta se puede producir miel en las casas. Cuando no existía la carretera, se producían las velas con una mezcla de su cera y la fibra de Heliocarpus donnell-smithii Rose (majagua).

- Melipona solani (Jach k'ojo' o Cojo). Para los mayas lacandones es la abeja "verdadera"; hacen la diferenciación con la abeja "extranjera", la Apis mellifera, la cual, de acuerdo con los pobladores, desplazó a las nativas. Melipona solani es una abeja parecida a Apis mellifera y puede estar cerca de las casas. Cuando no se conocía el azúcar, su miel se extraía de las colonias que se encontraban en la selva y servía como endulzante de bebidas, principalmente del pozol.

- Nannotrigona perilampoides (Bor kap). Aunque en términos generales la miel de esta abeja es del agrado de la población, su consumo es limitado debido a su poca producción.

- Paratrigona opaca (At sats). Es una abeja dócil, de la que no se extrae miel; hace sus nidos en árboles cuyo diámetro es reducido, de hasta 10 centímetros; pequeña, poco común y de color negro.

- Partamona bilineata (K'e'k'an). Es una abeja de comportamiento defensivo. Cuando es molestada se enreda en el cabello, "muerde" la cara o los brazos. La miel se consume raramente porque su sabor es amargo.

- Plebeia frontalis (Ajchi'; Ak chip kap). Son abejas que se ubican en huecos de troncos secos, producen poca miel. Su piquera tiene forma de boca, cuya entrada tiene una mezcla de cera y lodo.

- Plebeia llorentei (mejen kap'; yit mash). A nivel general, la población no la diferencia de la anterior, sólo aquellas personas de mayor edad que tienen la experiencia de hacer recorridos por la selva. Esta especie se caracteriza por hacer sus nidos en las copas de los árboles. Una característica distintiva es que chupa el sudor de las personas.

- Plebeia pulchra (mejen kap'; yit mash). Es una abeja pequeña, su nido lo hace en los huecos de los árboles, en partes altas; su miel se consume esporádicamente.

- Scaptotrigona pectoralis (Ak tun kap'; kan sak). Es una abeja con un comportamiento defensivo, "muerde" sobre todo en la cabeza. En comparación 
con las otras abejas nativas produce miel en buenas cantidades y gusta su sabor; se emplea para endulzar bebidas. Además, se reporta que su miel cura la tos. La cera se emplea para pegar las cuerdas que van amarradas a las flechas que venden como artesanía. Muchos nidos se pueden ubicar en árboles como Heliocarpus donnell-smithii Rose (majagua).

- Scaura argyrea (K'amas kap'). Es una abeja con la cual la población no tiene mucha relación porque construye su panal junto al de las termitas (termiteros). De acuerdo a lo expresado por la gente, esta abeja produce dos tipos de miel: para la reina y para las obreras. No se consume ningún tipo de miel; cuando se llega a consumir, y en exceso, puede ocasionar mareos e incluso vómitos.

- Tetragona mayarum (Yus). La miel es agradable al gusto de los mayas lacandones, pero no se consume mucho, debido al poco conocimiento que se tiene sobre su localización.

- Tetragonisca angustula (Ajyus). Es una abeja dócil, pequeña, de color amarillo con negro. Los nidos de esta abeja son hechos en pequeñas aberturas, incluso de paredes; la entrada de su nido por lo regular es una boquilla. Es una especie manejable, se puede producir en las casas, sobre todo en troncos. La miel se consume como endulzante y para curar la tos.

- Trigona corvina (Ajxi'p'). Es una abeja con comportamiento defensivo; es de color negro y de acuerdo con los mayas lacandones su cuerpo es resinoso. No se consume su miel. Los nidos son usualmente grandes y se ubican en los árboles.

- Trigona fulviventris (Ajmur; mu kap). Es una abeja que tiene su nido en la tierra y su miel no es muy apreciada; ésta se puede extraer escarbando como a un metro debajo de la tierra.

- Trigona nigerrima (Ajk'uk'is kap'). Es una abeja de color negro y tiene un olor no grato a los mayas lacandones; casi no se consume su miel. Su panal se ubica en los árboles.

Es importante señalar que el conocimiento de la población sobre las abejas es diferenciado de acuerdo a estratos de edad, y el poder identificarlas no implica que usen sus productos. El consumo de miel se orienta principalmente a ser endulzante y en algunas ocasiones medicinal. La tabla siguiente muestra el porcentaje del conocimiento que los mayas lacandones tienen sobre las abejas y para qué las ocupan (Tabla 2). 


\begin{tabular}{|c|c|c|c|c|c|c|}
\hline Nombre científico & $\begin{array}{l}\text { Nombre maya } \\
\text { lacandón }\end{array}$ & $\begin{array}{c}1 \\
\text { ¿Conoce } \\
\text { la abeja? } \\
(\%)\end{array}$ & $\begin{array}{c}2 \\
\text { ¿Usa los } \\
\text { productos } \\
\text { de la } \\
\text { colmena? } \\
(\%)\end{array}$ & $\begin{array}{c}3 \\
\text { Uso } \\
\text { alimenticio } \\
(\%)\end{array}$ & $\begin{array}{c}4 \\
\text { Uso } \\
\text { artesanal } \\
\text { (cera) (\%) }\end{array}$ & $\begin{array}{c}5 \\
\text { Alimentación } \\
\text { y artesanal } \\
\text { (cera) }(\%)\end{array}$ \\
\hline $\begin{array}{l}\text { Cephalotrigona } \\
\text { zexmeniae }\end{array}$ & K'anit & 51.5 & 25.7 & 88.9 & 0.0 & 11.1 \\
\hline Melipona solani & Jach K'ojo' & 85.3 & 37.9 & 72.7 & 4.5 & 22.7 \\
\hline $\begin{array}{l}\text { Nannotrigona } \\
\text { perilampoides }\end{array}$ & Bor kap & 4.4 & 0 & 0 & 0 & 0 \\
\hline Paratrigona opaca & At sats & 2.9 & 0 & 0 & 0 & 0 \\
\hline Partamona bilineata & K'e'k'an & 85.3 & 13.8 & 87.5 & 12.5 & 0.0 \\
\hline Plebeia frontalis & Ajchi' o Ak chip kap & 75.0 & 39.2 & 75.0 & 5.0 & 20.0 \\
\hline Plebeia llorentei & $\begin{array}{l}\text { Mejen kap, } \\
\text { yit mash }\end{array}$ & 61.7 & 30.9 & 92.3 & 0 & 7.7 \\
\hline Plebeia pulcra & $\begin{array}{c}\text { Mejen kap, } \\
\text { yit mash }\end{array}$ & 61.7 & 30.9 & 92.3 & 0 & 7.7 \\
\hline $\begin{array}{l}\text { Scaptotrigona } \\
\text { pectoralis }\end{array}$ & $\begin{array}{c}\text { Ak tun kap'; kan sak } \\
\text { (es rojo) }\end{array}$ & 58.8 & 30.0 & 91.7 & 0.0 & 8.3 \\
\hline Scaura argyrea & K'amas kap' & 76.5 & 42.3 & 90.9 & 4.5 & 4.5 \\
\hline Tetragona mayarum & Yus & 4.4 & 0 & 0 & 0 & 0 \\
\hline $\begin{array}{l}\text { Tetragonisca } \\
\text { angustula }\end{array}$ & Ajyus & 95.6 & 46.2 & 83.3 & 10.0 & 6.7 \\
\hline Trigona corvina & Ajxi'p' & 51.5 & 22.9 & 75.0 & 25.0 & 0.0 \\
\hline Trigona fulviventris & $\begin{array}{l}\text { Murish kap, Pupus } \\
\text { kap, Ajmur, mu kap }\end{array}$ & 48.5 & 15.2 & 80.0 & 20.0 & 0.0 \\
\hline Trigona nigerrima & Ajk'uk'is kap' & 54.4 & 24.3 & 100.0 & 0.0 & 0.0 \\
\hline
\end{tabular}

Tabla 2. Especies de abejas nativas que conoce la gente de Nahá y los porcentajes de las personas que les dan uso: 1 , se explica el porcentaje total de las personas entrevistadas que conocen a cada una de las abejas; 2 , De las personas que conocen la abeja, se calcula el porcentaje de quienes la usan; 3, 4 y 5, De quienes usan productos de la abeja, se determinó el porcentaje que lo usan para alimentación, artesanal (cera) y alimentación y artesanal

(Trabajo de campo, octubre 2015 a enero 2018).

\section{Conocimiento por estrato de edades}

El presente apartado describe la relación que existe entre el conocimiento que tienen los pobladores sobre las abejas nativas y los estratos diferenciados por edades. La encuesta aplicada arrojó que de forma general las principales especies de abejas nativas sin aguijón que conocen los mayas lacandones de Nahá son Tetragonisca angustula (95.5\%), Melipona solani (85.2\%), Scaura argyrea (76.4\%) y Plebeia frontalis (75\%) (Grafica 1). 


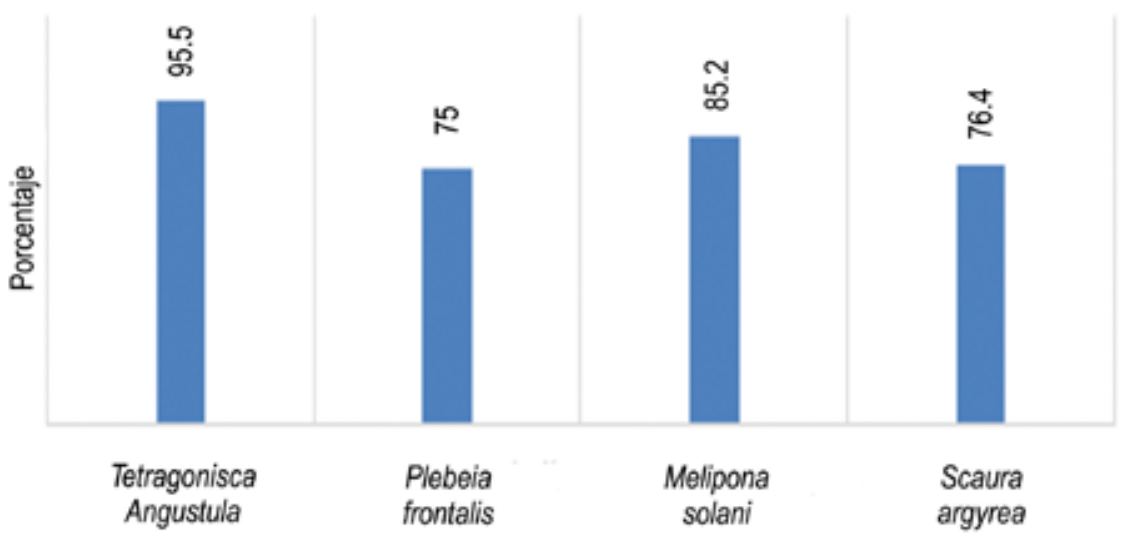

Nombre de las principales especies de abejas

Gráfica 1. Porcentaje de encuestados que conocen las cuatro principales especies de meliponinos.

Llama la atención que, aunque Melipona solani es considerada como la abeja "verdadera", no sea la que más conozcan ni la que más usen; es probable que la razón sea que su manejo no es sencillo, por ser una abeja sensible (Arnold et al., 2018a). La especie más aprovechada es Tetragonisca angustula, cuya adaptación a diferentes hábitats contribuye a que sus panales se ubiquen tanto en la selva como en la comunidad.

El conocimiento que tiene la población sobre las abejas es diferenciado y se relaciona con los estratos de edades; las personas del estrato III (46-80 años) prácticamente conocen las cuatro principales abejas, a diferencia del estrato I (18-30 años), cuyo conocimiento es menor en todas las especies, excepto en Tetragonisca angustula. Las actividades diferenciadas de los diversos estratos de edad influyen en el conocimiento y aprovechamiento de los productos de las abejas. Los jóvenes (estrato I) están incorporados a trabajos que implican un apoyo económico, como proyectos de conservación y productivos auspiciados por el gobierno y onGs, mientras que las personas de más edad (estrato III) se dedican a actividades orientadas a la agricultura, caza, pesca y recolección; por tanto, ello les permite estar en constante relación con los diferentes tipos de vegetación que hay en la comunidad.

A nivel general los datos obtenidos muestran que, aunque la gente conozca a las abejas no significa que las aprovechen y menos que exista un manejo. Del $100 \%$ de las personas que conocen las cuatro especies de abejas no se alcanza ni el 50\% de quienes ocupan alguno de sus productos: Tetragonisca angustula (46\%), Scaura argyrea (42.3\%), Plebeia frontalis (40\%) y Melipona solani (38\%) (Grafica 2). 


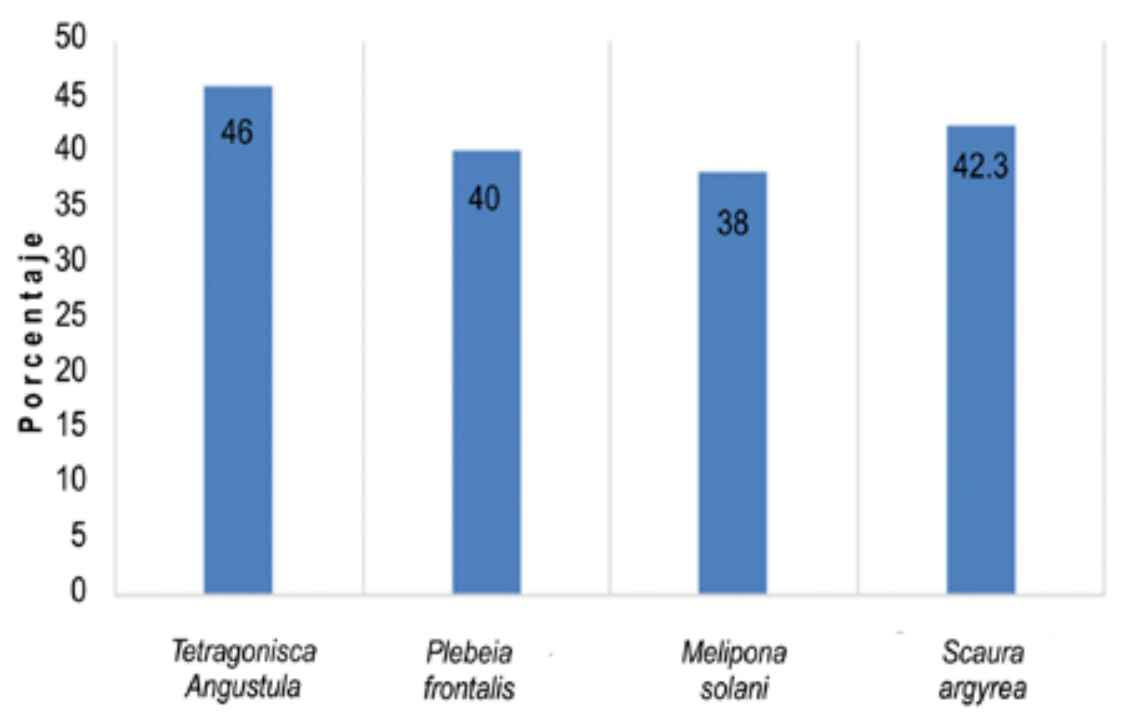

Especies de abejas más usadas

Gráfica 2. Porcentaje de personas que usan las cuatro especies de abejas más reconocidas.

El conocimiento de abejas por estrato de edad es diferenciado; en el estrato I (18 a 30 años) la gente conoce a las abejas en los porcentajes siguientes: Tetragonisca angustula (96\%), Melipona solani (75\%), Scaura argyrea (67\%) y Plebeia frontalis (53\%). En el estrato II (31 a 45 años) conocen Tetragonisca angustula, Melipona solani y Plebeia frontalis en un $89 \%$, mientras que para Scaura argyrea es del $69 \%$; esta especie es la menos conocida en los estratos uno y dos, lo cual posiblemente se deba a que sus patrones de comportamiento están asociados a compartir espacios con las termitas y por tanto no es tan fácil observarlas. Finalmente, para el estrato III (46 a 80 años), todas las personas encuestadas conocen el nombre maya de Tetragonisca angustula (100\%), Melipona solani (100\%), Scaura argyrea (100\%) y Plebeia frontalis (100\%) (Gráfica 3). Esta diferencia en el conocimiento de cada uno de los estratos se debe fundamentalmente a que las personas de mayor edad (estrato III) trabajan más en el campo que los jóvenes, quienes realizan trabajos institucionales impulsados por la reserva.

El conocimiento de las abejas no implica su utilización. En el estrato I (18 a 30 años) aprovechan a las abejas en los porcentajes siguientes: Tetragonisca angustula (51\%), Scaura argyrea (47\%), Plebeia frontalis (47\%) y Melipona solani (33\%). En el estrato II (31 a 45 años) son dos las especies que se aprovechan por más del 50\% de los encuestados: Scaura argyrea (53\%) y Melipona solani (52\%); mientras que las otras dos se aprovechan en un porcentaje menor: Tetragonisca angustula 


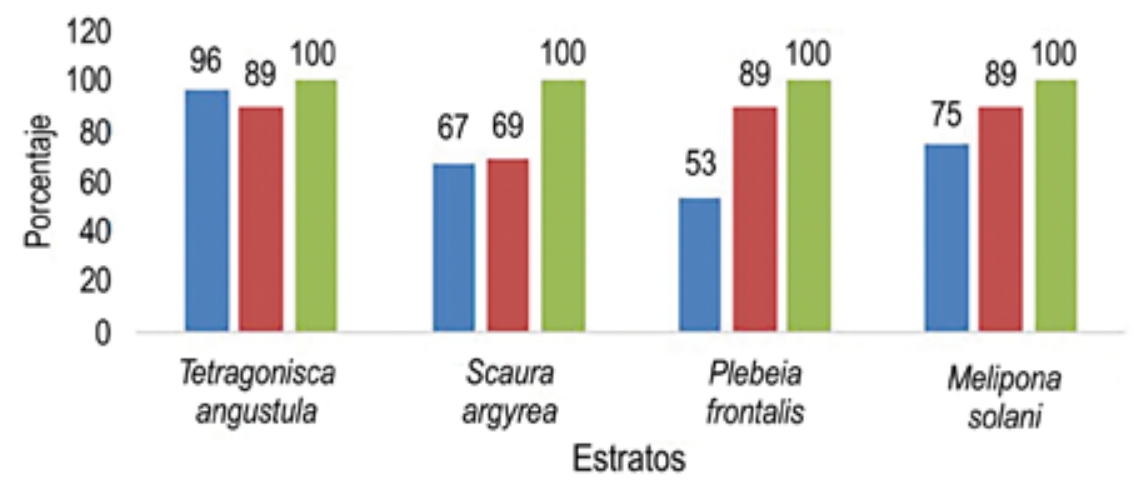

\section{घEstrato $1(18-30) \quad$ EEstrato $2(31-45) \quad$ =Estrato $3(46-80)$}

Gráfica 3. Porcentaje por estrato de edad que conocen las principales abejas.

(38\%) y Plebeia frontalis (33\%). Finalmente, para el estrato III (46 a 80 años), llama la atención que ningún producto de abeja es ocupado más allá del $50 \%$ de los entrevistados: Tetragonisca angustula (41\%), Scaura argyrea (29\%), Plebeia frontalis (35\%) y Melipona solani (31\%). Esto significa que, aunque las personas más adultas conocen las abejas, su aprovechamiento es reducido, lo cual se pudiera deber al respeto que se tiene sobre su cuidado o a la normatividad restrictiva que tiene la reserva en relación con las prácticas culturales que realizan. En este sentido, los mecanismos de reproducción del conocimiento son limitados con las generaciones más jóvenes (Gráfica 4).

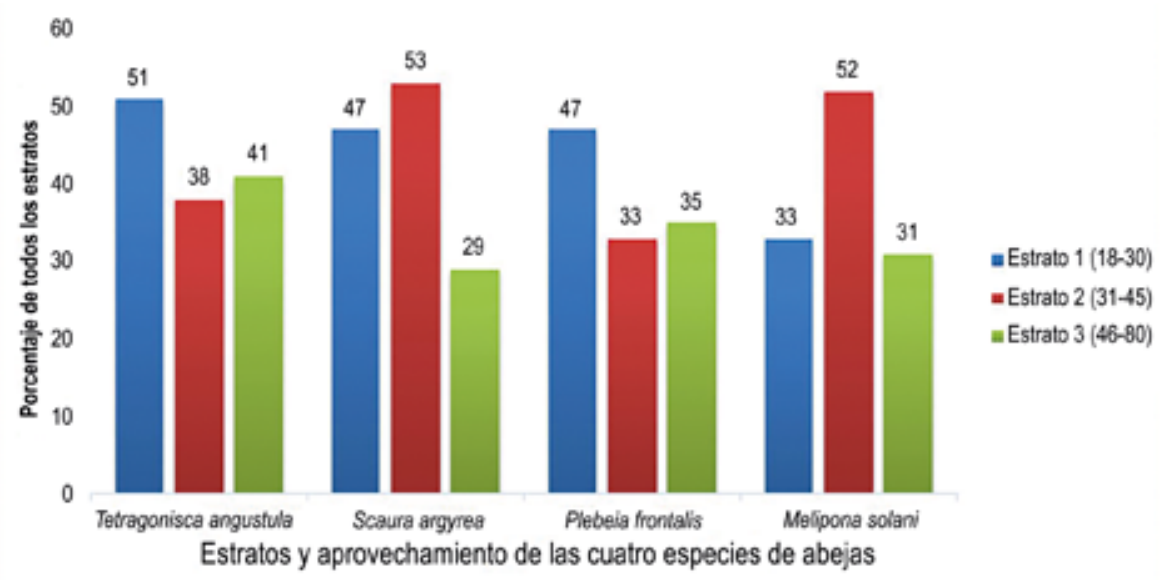

Gráfica 4. Porcentaje por estrato de personas que aprovechan algún producto de las cuatro principales especies de abejas conocidas. 
El manejo de poblaciones de ANSA era una práctica desarrollada hace algunos años, pero se ha ido perdiendo. Actualmente, y en general para toda la población, es más común la extracción que el manejo. Además, se observa que el consumo de la miel disminuye debido a la existencia de productos que la sustituyen, principalmente azúcar y medicamentos alópatas. Es más barato comprar los productos que emplear el tiempo para buscar miel por la selva. El impacto de las políticas ecológicas también es un factor a considerarse porque al decretarse el territorio de Nahá como reserva se prohibieron prácticas en el manejo de los bienes naturales, lo cual tiene impacto sobre la reproducción de los conocimientos.

\section{Conclusiones}

De acuerdo con los datos encontrados existen 15 especies de meliponinos que identifican los mayas lacandones, pero que son conocidas de forma distinta según las diferentes edades de los pobladores. Las personas mayores identifican más abejas nativas que las jóvenes, diferencia que refleja por un lado que los mecanismos de transmisión intergeneracional no son eficaces y por el otro que la influencia del Área Natural Protegida constituida a partir de 1998 implementa una normativa tendiente a la restricción de ciertas prácticas relacionadas con el manejo de los bienes naturales; este hecho promueve la disminución del contacto de la gente con la selva, o en su caso orienta las acciones hacia proyectos y programas que promueve la institución. En este sentido, cuando no existía el ANP —antes de 1998- el contacto de los mayas lacandones con su entorno ecológico era más intenso; obligaba a la gente a un conocimiento más amplio y preciso sobre las dinámicas de uso y manejo de los bienes naturales.

El financiamiento de proyectos de desarrollo es uno de los factores que orientan a la población a realizar actividades que son promovidas por las instituciones, de modo que muchas prácticas tradicionales son abandonadas y por lo tanto olvidadas. En este sentido, la población en general aprovecha las ANSA de forma marginal. Aunque la gente de mayor edad identifica más a las abejas en comparación con los jóvenes no significa que realicen un aprovechamiento y menos aún un manejo de las abejas nativas.

El desarrollo de actividades económicas, motivadas por proyectos de desarrollo impulsadas por instituciones gubernamentales u organizaciones civiles que contribuyen al incremento del ingreso económico en las familias, es un factor importante que contribuye a que la población dedique más tiempo a las actividades que les son propuestas por ellas que al manejo de sus bienes naturales.

Finalmente, el hecho que los mayas lacandones reconozcan e incluso designen a algunas abejas nativas con diferentes nombres y otras las caractericen de acuerdo con su comportamiento, al tipo de nido que construyen o a su tamaño, refleja que la relación con tales insectos es compleja. En la medida en que las prácticas de uso y manejo disminuyen en torno a las ANSA, el resultado es induda- 
blemente la pérdida de conocimientos y saberes, ejes clave para la conservación de la diversidad de las abejas. En este sentido, la vulnerabilidad de las prácticas orientadas hacia la conservación de las ANSA se ven mermadas y con ello el patrimonio biocultural que pertenece a la sociedad mexicana, pero en particular a los mayas lacandones de Nahá.

\section{Agradecimientos}

A Chankín Atanacio López (†) y a Juan José Chankín Elías, por su guía y ayuda en la selva de Nahá; sin él mucha de la información registrada en este artículo no hubiera sido posible.

\section{Bibliografía}

Aldasoro Maya Elda Miriam

2009 "Etnoentomología”, La diversidad biológica del Estado de México. Estudio de estado, pp. 299-304, Gerardo Ceballos, Rurik List, Gloria Garduño, Rubén López Cano, María José Muñozcano, Enrique Collado, Jaime Eivin y San Román (comps.). Estado de México: Biblioteca Mexiquense del Bicentenario (Colección Mayor).

Aldasoro Maya, Elda Miriam, Noemí Arnold y Corina Yuriet Burguete Rosales

2015 "Los meliponinos de Comalcalco, Tabasco, una primera aproximación desde el enfoque biocultural", ponencia presentada en el IX Congreso de Etnobiología, celebrado en San Cristóbal de Las Casas, Chiapas, del 27 de abril al 2 de mayo de 2014.

Aldasoro Maya, Elda Miriam, Teresita Avilez López, Gabriela Vera Cortés y Johannes Van der Wal

2016 "Saberes contemporáneos, meliponinos y escalamiento de la agroecología: reconociendo paisajes culturales", ponencia presentada en el X Congreso Mexicano de Etnobiología, Mérida, Yucatán, del 19 al 23 de septiembre de 2016.

Arnold, Noemi, Raquel Zepeda, Marco Antonio Vásquez Dávila y Elda Miriam Aldasoro Maya 2018a Las abejas sin aguijón y su cultivo en Oaxaca, México. Con catálogo de especies. San Cristóbal de Las Casas: El Colegio de la Frontera Sur, Comisión Nacional para el Conocimiento y Uso de la Biodiversidad.

Arnold, Noemi, Ricardo Ayala, Jorge Mérida, Philippe Sagot, Elda Miriam Aldasoro Maya y Rémy Vandame

2018b "Nuevos registros de abejas sin aguijón (Apidae: Meliponini) para los estados de Chiapas y Oaxaca, México", Revista Mexicana de Biodiversidad, 89: 651-665. Dol: https://doi.org/10.22201/ib.20078706e.2018.3.2429. 
Ayala, Ricardo

1999 "Revisión de las abejas sin aguijón de México (Hymenoptera: Apidae: Meliponini)”, Folia Entomológica Mexicana, 106: 1-123.

Ayala, Ricardo, Víctor H. González y Michael S. Engel

2013 "Mexican Stingless Bees (Hymenoptera: Apidae): Diversity, Distribution, and Indigenous Knowledge", Pot-Honey: A Legacy of Stingless Bees, pp. 135-152, Patricia Vit, Silvia Pedro y David Roubik (eds.). Nueva York: Springer.

Baer, Phillip y William Merrifield

1972 Los Lacandones de México. México: Instituto Nacional Indigenista.

Boremanse, Didier

1978 "The Social Organization of the Lacandon Indians of Mexico: A Comparative Study of Two Maya Forest Peoples", tesis de doctorado en Antropología Social. Oxford: Universidad de Oxford.

Cano Contreras, Eréndira Juanita, Chrystian Martínez Martínez y Carlos César Balboa Aguilar 2013 "Abeja de monte" (Insecta: Apidae, meliponini) de los choles de Tacotalpa, Tabasco: conocimiento local, presente y futuro”, Etnobiología, 11 (2): 4757.

Chan Mutul, Guelmy, Gabriela Vera Cortés, Elda Miriam Aldasoro Maya y Laura Sotelo Santos 2019 "Retomando saberes contemporáneos. Un análisis del panorama actual de la meliponicultura en Tabasco", Estudios de Cultura Maya, LIII: 289-326. DoI: https://doi.org/10.19130/iifl.ecm.2019.53.947.

Comisión Nacional de Áreas Naturales Protegidas (CONAnP)

2006 Programa de conservación y manejo. Área de protección de flora y fauna Nahá. México: Comisión Nacional de Áreas Naturales Protegidas.

Contreras Cortés, Ulises

2018 "Abejas nativas sin aguijón (Apidae: Meiponini) registradas para el estado de Chiapas", Etnobiología de Chiapas. Disciplinas raíces de la etnobiologia. Tomo II, pp. 211-234, Cecilia Elizondo, Ramón Mariaca, Fausto Bolom Ton (eds.). San Cristóbal de Las Casas: Chiapaneros.

Contreras Cortés, Ulises, Ramón Mariaca y Miguel Ángel Pérez

2015 "El proceso de sucesión ecológica entre los lacandones de Nahá, Chiapas, México", Etnobiología, 13 (2): 49-62.

Contreras Cortés Ulises y Ramón Mariaca Méndez

2016 Manejo de los recursos naturales entre los mayas lacandones de Nahá. San Cristóbal de Las Casas, Chiapas: El Colegio de la Frontera Sur.

Echazarreta, Carlos, Javier J. G. Quezada Euán, Luis M. Medina y Katherine L. Pasteur 1997 "Beekeeping in the Yucatan Peninsula: Development and Current Status", Bee World, 78 (3): 115-127. Dol: https://doi.org/10.1080/000577 2X.1997.11099346. 
Eroza, Enrique

2006 Lacandones. México: Comisión Nacional para el Desarrollo de los Pueblos Indígenas.

García Amaro, Enriqueta

2003 "Distribución de la precipitación en la República Mexicana. Investigaciones geográficas”, Boletín del Instituto de Geografía, 50: 67-76.

González Acereto, Jorge Alberto

2012 "La importancia de la meliponicultura en México, con énfasis en la Península de Yucatán”, Bioagrociencias, 5 (1): 34-41.

Guzmán, Miguel, Carlos Balboa, Remy Vandame, María Luisa Albores y Jorge González Acereto

2011 Manejo de las abejas nativas sin aguijón en México. Melipona beecheii y Scaptotrigona mexicana. San Cristóbal de Las Casas: El Colegio de la Frontera Sur.

Halffter, Gonzalo

1978 "Un nuevo patrón de dispersión en la zona de transición mexicana: el mesoamericano de montaña”, Folia Entomológica Mexicana, 39-40: 219-222.

1987 "Biogeography of the Montane Entomofauna of Mexico and Central America”, Annual Review of Entomology, 32: 95-114.

2003 "Biogeografía de la entomofauna de montaña de México y América Central", Una perspectiva latinoamericana de la biogeografía, pp. 87-97, J. J. Morrone y J. Llorente (eds.). México: Universidad Nacional Autónoma de México, Facultad de Ciencias.

Instituto de Historia Natural

2000 Propuesta de programa de manejo para las áreas de protección de flora y fauna Nahá y Metzabok. Tuxtla Gutiérrez, Chiapas: Secretaría de Medio Ambiente, Recursos Naturales y Pesca.

Kremen Claire, Neal M. Williams, Marcelo A. Aizen, Barbara Gemmill-Herren, Gretchen LeBuhn, Robert Minckley, Laurence Packer, Simon G. Potts, T'ai Roulston, Ingolf SteffanDewenter, Diego P. Vázquez, Rachael Winfree, Laurie Adams, Elizabeth E. Crone, Sarah S. Greenleaf, Timothy H. Keitt, Alexandra Maria Klein, James Regetz y Taylor H. Ricketts

2007 "Pollination and Other Ecosystem Services Produced by Mobile Organisms: A Conceptual Framework for the Effects of Land-Use Change", Ecology Letters, 10: 299-314. DoI: https://doi.org/10.1111/j.1461-0248.2007.01018.x.

Marion Singer, Marie Odile

1999 El poder de las hijas de luna. México: Consejo Nacional para la Cultura y las Artes, Instituto Nacional de Antropología e Historia, Plaza y Valdés.

Michener, Charles Duncan

2007 The Bees of the World. Maryland: The Johns Hopkins University.

2013 "The Meliponini”, Pot-Honey. A Legacy of Stingless Bees, pp. 3-17, Patricia Vit, Silvia Pedro y David Roubik (eds.). Nueva York: Springer. 
Miranda, Faustino y Efraín Hernández

1963 "Los tipos de vegetación de México y su clasificación”, Boletín de la Sociedad Botánica de México, 28: 29-179. Dol: https://doi.org/10.17129/botsci.1084.

Murillo, Rosa María

1984 "Uso y manejo actual de las colonias de Melipona beecheii (Apidae, Meliponinae) en el Estado de Tabasco, México”, Biótica, 9 (4): 422-428.

Padilla Vargas, Pavel Jairo, Marco Antonio Vásquez Dávila, Tania G. García Guerra y María Luisa Albores González

2014 "Pisilnekmej: una mirada a la cosmovisión, conocimientos y prácticas nahuas sobre Scaptotrigona mexicana en Cuetzalan, Puebla, México", Etnoecológica, 10: 37-40.

Palacio, José Luis, María Teresa Sánchez, José María Casado, Enrique Propin, Javier Delgado, Alejandro Velázquez, Luis Chlas, María Inés Ortiz, Jorge González, Gerardo Negrete, Josefina Gabriel y Roberto Márquez

2004 Indicadores para la caracterización y el ordenamiento territorial. México: Secretaría de Medio Ambiente y Recursos Naturales, Instituto Nacional de Ecología, Universidad Nacional Autónoma de México, Secretaría de Desarrollo Social.

Pat Fernández, Lucio Alberto, Francisco Anguebes, Juan Pat, Pablo Hernández y Rodimiro Ramos

2018 "Condición y perspectivas de la meliponicultura en comunidades mayas de la reserva de la biósfera Los Petenes, Campeche, México", Estudios de Cultura Maya, LIII: 227-254. DoI: https://doi.org/10.19130/iifl.ecm.2018.52.939.

Patlán Martínez, Elia y José Trinidad Kanetas Ortega

2014 "Conservación y reproducción de las abejas sin aguijón (Scaptotrigona mexicana): tradición milenaria de la relación hombre naturaleza como práctica de identidad cultural”, ponencia presentada en el II Congreso Internacional y XVI Congreso Nacional de Ciencias Agronómicas. Universidad Autónoma de Chapingo, Texcoco, del 23 al 25 de abril de 2014.

Reyes-González, Alejandro, Andrés Camou Guerrero y Salvador Gómez Arreola

2016 "From Extraction to Meliponiculture: A Case Study of the Management of Stingless Bees in the West-Central Region of Mexico", Beekeeping and Bee Conservation: Advances in Research, pp. 201-223, Emerson Chambó (ed.). Amazonas: Universidad Federal del Amazonas, Instituto de Naturaleza y Cultura.

Sotelo Santos, Laura Elena, María Elena Guerrero y Carlos Álvarez

2012 "El cultivo tradicional de la abeja Melipona beecheii. Una constante en el huerto familiar entre los mayas de Yucatán”, El huerto familiar del sureste de México, pp. 293-322, Ramón Mariaca (ed.). Villahermosa, San Cristóbal de Las Casas, México: Secretaría de Recursos Naturales y Protección Ambiental del Estado de Tabasco, El Colegio de la Frontera Sur. 
Villanueva Gutiérrez, Rogel, David W. Roubik y Wilberto Colli Ucán

2005 "Extinction of Melipona beecheii and Traditional Beekeeping in the Yucatán

Peninsula”, Bee World, 86 (2): 35-41.

Leonardo Ernesto Ulises Contreras Cortés. Mexicano. Ingeniero Agrónomo especialista en Sociología Rural por la Universidad Autónoma Chapingo, maestro en Ciencias por El Colegio de la Frontera Sur y doctor en Ciencias por el Colegio de Posgraduados, campus Puebla. Adscrito a la Facultad de Ciencias Sociales de la Universidad Autónoma de Chiapas. Su principal línea de investigación es la Etnoecología de los mayas lacandones del sur y su proyecto en curso se titula "Conocimiento local de las abejas nativas de los mayas lacandones del norte, Ocosingo, Chiapas". Entre sus últimas publicaciones se encuentran "La etnoecología de los mayas lacandones. Una aproximación a los hach winik", "Abejas nativas sin aguijón (Apidae Meiponini) registradas para el estado de Chiapas" e "Importancia y uso de las palmas entre los mayas lacandones de Nahá, Chiapas", este último en coautoría.

mtroulises@hotmail.com

Amparo Vázquez García. Mexicana. Licenciada en Biología por el Instituto Superior de Zacapoaxtla, realizó estudios de maestría en Educación por la Universidad Campesina en Red (UCIRED). Adscrita a la Universidad Intercultural del Estado de Puebla. Sus especialidades son los hongos silvestres útiles y las abejas nativas de zonas tropicales. Su proyecto en curso se titula "Conocimiento local de las abejas nativas de los mayas lacandones del norte, Ocosingo, Chiapas". Su publicación más reciente se titula "Etnomicología y venta de hongos en un mercado del noroeste del estado de Puebla”, en coautoría.

ampa_20@hotmail.com

Elda Miriam Aldasoro Maya. Mexicana. Licenciada en Biología por la Facultad de Estudios Superiores Iztacala de la Universidad Nacional Autónoma de México, maestra y doctora en Antropología Ambiental por la Universidad de Washington. Adscrita a El Colegio de la Frontera Sur, unidad Villahermosa. Su principal línea de investigación es la masificación de la agroecología. Actualmente desarrolla los siguientes proyectos: "La meliponicultura como herramienta pedagógica para el escalamiento de la agroecología", "Huertos escolares agroecológicos y bioculturales en Villahermosa, Tabasco, como instrumento para la masificación de la agroecología" y "La pedantropología como propuesta para la masificación agro- 
ecológica". Entre sus publicaciones más recientes se encuentran "Registros nuevos de abejas sin aguijón (Apidae: Meliponini) para los estados de Chiapas y Oaxaca, México", "Retomando saberes contemporáneos. Un análisis del panorama actual de la meliponicultura en Tabasco" y "Agroecology on the Periphery: A Case from the Maya-Achí Territory, Guatemala", todos en coautoría.

miriam.aldasoro@gmail.com

Jorge Mérida Rivas. Mexicano. Ingeniero biotecnólogo por la Universidad Autónoma de Chiapas, maestro en Ciencias en Recursos Naturales y Desarrollo Rural con orientación en entomología tropical por El Colegio de la Frontera Sur. Actualmente realiza estudios de doctorado en el Programa de Doctorado en Ciencias en Ecología y Desarrollo Sustentable en El Colegio de la Frontera Sur, unidad San Cristóbal de Las Casas. Su principal línea de investigación es la conservación de la biodiversidad. Su proyecto actual se titula "Revisión del género Xylocopa Latreille, 1802 (Hymenoptera, Apinae, Xylocopinae) de México y Centroamérica y filogenia del subgénero Xylocopoiddes". Entre sus publicaciones recientes están "Quaternary Climate Instability is Correlated with Patterns of Population Genetic Variability in Bombus huntii", "Recent Low Levels of Differentiation in the Native Bombus ephippiatus (Hymenoptera: Apidae) along Two Neotropical Mountain-Ranges in Guatemala" y "Registros nuevos de abejas sin aguijón (Apidae: Meliponini) para los estados de Chiapas y Oaxaca, México", todos en coautoría.

jorgejoalmer@gmail.com 